\title{
Distributed Containment Control of Networked Fractional-Order Systems with Delay-Dependent Communications
}

\author{
Xueliang Liu, ${ }^{1,2}$ Bugong $X u^{1,2}$ and Lihua $X e^{3}$ \\ ${ }^{1}$ College of Automation Science and Engineering, South China University of Technology, \\ Guangzhou 510640, China \\ ${ }^{2}$ Key Laboratory of Autonomous Systems and Network Control, Ministry of Education, \\ Guangzhou 510640, China \\ ${ }^{3}$ School of Electrical and Electronic Engineering, Nanyang Technological University, Singapore 639798
}

Correspondence should be addressed to Bugong Xu, aubgxu@scut.edu.cn

Received 5 March 2012; Accepted 26 May 2012

Academic Editor: Baocang Ding

Copyright (C) 2012 Xueliang Liu et al. This is an open access article distributed under the Creative Commons Attribution License, which permits unrestricted use, distribution, and reproduction in any medium, provided the original work is properly cited.

This paper is concerned with a containment problem of networked fractional-order system with multiple leaders under a fixed directed interaction graph. Based on the neighbor rule, a distributed protocol is proposed in delayed communication channels. By employing the algebraic graph theory, matrix theory, Nyquist stability theorem, and frequency domain method, it is analytically proved that the whole follower agents will flock to the convex hull which is formed by the leaders. Furthermore, a tight upper bound on the communication time-delay that can be tolerated in the dynamic network is obtained. As a special case, the interconnection topology under the undirected case is also discussed. Finally, some numerical examples with simulations are presented to demonstrate the effectiveness and correctness of the theoretical results.

\section{Introduction}

In recent years, coordination of multiagent systems has attracted considerable interest in the control community due to their wide application areas in formation control [1-3], flocking/swarming [4,5], consensus [6-9], sensor networks [10, 11], synchronization of complex networks [12,13], and distributed computation [14]. A common character of these applications is that each individual agent lacks a global knowledge of the whole system and can only send and/or obtain state information from its neighbors through local communications. Significant progress has been made in the coordination problem (see, e.g., $[11,15,16]$ and the references therein). 
As a special case of coordination control, containment control means to drive the followers to be in the convex hull which is spanned by the leaders. Different from general leader-following consensus problems, where there exist multiple leaders and multiple followers in containment problems. The coordinate objective is likely to be one or several target sets in multiagent coordination control. These target sets may be a biological communities, a team of robots, a food enrichment area, and so on. For example, a kind of biological group hunts another kind of biological communities, a team of biological group cooperatively builds their nest, and several agents lead a team of agents avoiding hazardous obstacles. Thus, how to control a multiagent system to achieve a common target becomes an interesting problem.

The idea of fractional calculus has been known since the development of the regular calculus, with the first reference probably being dated back to the seventeenth century [17], where the meaning of derivative of order one-half was first mentioned. Although it has a long history, the fractional calculus applications to physics and engineering are just a recent focus of interest. Moreover, fractional derivatives provide an excellent tool for the description of memory and hereditary properties of various materials and processes. As pointed out by many researchers, many physical systems are more suitable to be modeled by fractional-order dynamic equations [18]. Many systems are known to display fractional-order dynamics, such as viscoelastic systems, electromagnetic waves, and quantum evolution of complex systems. In additional, integer-order systems can be regarded as a special case of fractional-order systems.

More recently, many interesting agent-related consensus problems are under investigation, and fractional-order consensus becomes a hot topic. The consensus problem of fractional-order systems is first proposed and investigated by Cao et al. [19, 20], where three different cases of coordination models are introduced. By employing a varyingorder fractional coordination strategy, a higher convergence performance is obtained. Sun et al. [21] study the consensus problem for fractional-order systems under undirected scale-free networks. They also compared the convergence rate of fractional-order dynamics and the integer-order dynamics. In order to increase the convergence speed and ensure the exponential convergence, a switching order consensus protocol is employed. Shen et al. [22, 23] consider the consensus problem of fractional-order systems with nonuniform input and/or communication delays over directed networks. Based on the Nyquist stability criterion and frequency domain approach, some sufficient conditions are obtained to ensure the fractional-order consensus. Formation control problems for fractional-order systems were discussed in [24]. However, little research work has been done toward the problem of containment control of fractional-order system, which is the main focus of this paper.

Motivated by the above discussion, in this paper, we consider the containment problem of networked fractional-order systems over directed topologies. Different from generally leader-following consensus problem, there exist multiple leaders in a containment control problem. The objective is to drive the followers to be in the convex hull formed by the leaders. On the other hand, in practice delays unavoidably exist due to the finite speed of transmission, acquisition, and traffic congestions. Therefore, studying the agents with the form of fractional-order dynamics over delayed communication channel becomes very significant.

The rest of the paper is organized as follows. Section 2 gives some preliminaries on algebraic graph theory and Caputo fractional operator and formulates the problem under investigation. In Section 3, containment control under fixed directed topologies and delayed 
communication channels is investigated. In Section 4, two examples are provided to verify the theoretical analysis. Finally, some concluding remarks will be drawn in Section 5.

Some remarks on the notation are given as follows: $[\alpha]$ stands for the integer part of $\alpha, \mathbf{1}_{n}$ is an $n$-dimension column vector with all ones. $I_{N}$ is the identity matrix with dimension $N \times N . \mathbb{R}^{N \times N}$ and $\mathbb{R}^{N}$, respectively, denote the family of all $N \times N$ dimensional matrices and the $N$ dimensional column vector. The notation $A>0(A \geq 0)$ means that matrix $A$ is positive definite (semidefinite). " $\|x\|$ " stands for the Euclidean norm of vector $x . \lambda_{i}(A)$ and $\rho(A)$ denote the $i$ th eigenvalue of matrix $A$ and spectral radius of matrix $A$, respectively. diag $\{\cdots\}$ stands for a block diagonal matrix. $A \otimes B$ means the Kronecker product of matrices $A$ and $B$. A set $\Theta \in \mathbb{R}^{N}$ is convex if the line segment between any two elements in $\Theta$ lies in $\Theta$, that is, if for any $x, y \in \Theta$ and any $\gamma$ with $0 \leq \gamma \leq 1$, we have $\gamma x+(1-\gamma) y \in \Theta$. Similarly, a vector sum $\gamma_{1} x_{1}+\gamma_{2} x_{2}+\cdots+\gamma_{n} x_{n}$ is called a convex combination of $x_{1}, x_{2}, \ldots, x_{n}$ if the coefficients satisfy $\gamma_{i} \geq 0$ and $\sum_{i=1}^{n} \gamma_{i}=1$. The convex hull of $\Theta$ denoted by $\operatorname{co}\{\Theta\}$ is the intersection of all convex sets containing $\Theta$. For nonempty set $E$, the Euclid distance between point $x$ and set $E$ is defined as $d_{E}(x)=\inf _{y \in E}\|x-y\| . J=\{1,2, \ldots, N\}$.

\section{Preliminary}

Before formulating our problem, we introduce some basic concepts in graph theory and the Caputo fractional operator for fractional-order networks.

\subsection{Algebraic Graph Theory}

Algebraic graph theory is a natural framework for analyzing coordination problems. Let the interaction topology of information exchanged between $N$ agents be described by a directed graph $\mathcal{G}=\{\mathcal{V}, \mathcal{E}, \mathcal{A}\}$, where $\mathcal{V}=\{1,2, \ldots, N\}$ is the set of vertices, vertex $i$ represents the $i$ th agent, $\varepsilon \subset \mathcal{V} \times \mathcal{V}$ is the set of edge. An edge in $\mathcal{G}$ is denoted by an ordered pair $(j, i)$, representing that agent $i$ can receive information from agent $j$. The neighborhood of the $i$ th agent is denoted by $\mathcal{N}_{i}=\{j \in \mathcal{V} \mid(j, i) \in \mathcal{E}\} . \mathcal{A}=\left[a_{i j}\right]_{N \times N} \in R^{N}$ is called the weighted adjacency matrix of $\mathcal{G}$ with nonnegative elements where $a_{i i}=0$ and $a_{i j} \geq 0$ with $a_{i j}>0$ for $j \in \mathcal{N}_{i}$. The in-degree of agent $i$ is defined as $\operatorname{deg}_{\text {in }}(i)=\sum_{j=1}^{N} a_{i j}$, and the out-degree of agent $i$ is defined as $\operatorname{deg}_{\text {out }}(i)=\sum_{j=1}^{N} a_{j i}$. The Laplacian matrix of $\mathcal{G}$ is defined as $L=\boldsymbol{D}-\boldsymbol{A}$, where $\Phi=\operatorname{diag}\left\{\operatorname{deg}_{\text {in }}(1), \operatorname{deg}_{\text {in }}(2), \ldots, \operatorname{deg}_{\text {in }}(N)\right\}$. A sequence of edges $\left(i_{1}, i_{2}\right),\left(i_{2}, i_{3}\right), \ldots,\left(i_{k-1}, i_{k}\right)$ is called a path from agent $i_{1}$ to agent $i_{k}$. A directed tree is a directed graph, where every agent has exactly one neighbor except one agent has no neighbors. A spanning tree of $\mathcal{G}$ is a directed tree whose vertex set is $\mho$ and whose edge set is a subset of $\mathcal{E}$. In undirected graphs, if there is a path between any two vertices of a graph $\mathcal{G}$, then $\mathcal{G}$ is connected, otherwise disconnected.

\subsection{Caputo Fractional Operator}

For an arbitrary real number $\alpha$, the Riemann-Liouville and Caputo fractional derivatives are defined, respectively, as

$$
\begin{gathered}
{ }_{a} D_{t}^{\alpha} f(t)=\frac{1}{\Gamma(m-\alpha)} \frac{d^{m}}{d t^{m}} \int_{a}^{t}(t-\tau)^{m-\alpha-1} f(\tau) d \tau, \\
{ }_{a}^{C} D_{t}^{\alpha} f(t)=\frac{1}{\Gamma(m-\alpha)} \int_{a}^{t} \frac{f^{m}(\tau)}{(t-\tau)^{\alpha+1-m}} d \tau,
\end{gathered}
$$


where $m=[\alpha]+1$ is the first integer which is not less than $\alpha$ and $\Gamma(\cdot)$ is the Euler's gamma function.

It is worth pointing out that the advantage of Caputo approach is that the initial conditions for fractional-order differential equations with Caputo derivatives take on the same form as that for integer-order differential equations. For details, please refer to [17]. Therefore, we will only use the Caputo fractional operator in this paper to model the system dynamics. For notational simplicity, we rewrite ${ }_{a}^{C} D_{t}^{\alpha} f(t)$ as $f^{(\alpha)}(t)$ in the rest of the paper.

\subsection{Laplace Transform}

In the following, we will introduce the Laplace transform of the caputo fractional derivative which will fascinate the development of the subsequent results:

$$
\mathcal{L}\left\{f^{(\alpha)}(t)\right\}= \begin{cases}s^{\alpha} F(s)-s^{\alpha-1} f(0), & \alpha \in(0,1] \\ s^{\alpha} F(s)-s^{\alpha-1} f(0)-s^{\alpha-2} \dot{f}(0), & \alpha \in(1,2] .\end{cases}
$$

\subsection{Problem Formulation}

Consider a networked fractional-order system consisting of $N+m$ agents, where $N$ agents labeled by $1,2, \ldots, N$ are referred to as the follower agents and the other agents labeled by $N+1, \ldots, N+m$ act as leaders of the team. The information interaction topology among $N$ following-agents is described by the graph $\mathcal{G}=(\mathcal{U}, \mathcal{E}, \mathcal{A})$, and the whole system involving $N+m$ agents is conveniently modeled by a weighted directed graph $\overline{\mathcal{G}}=(\overline{\mathcal{U}}, \bar{\varepsilon}, \bar{A})$ with $\overline{\mathcal{V}}=\{1,2, \ldots, N+m\}$ and $\bar{A}=a_{i j} \in \mathbb{R}^{(N+m) \times(N+m)}, i, j=1,2, \ldots, N+m$, where the lower block submatrix of order $N$ can be regarded as $\mathcal{A}$. In this paper, we regard the convex hull spanned by multiple leaders as a virtual leader. The graph $\overline{\mathcal{G}}$ has a spanning tree meaning that there exits a path from the virtual leader to every follower agent. In general, the dynamic of each leader is independent of the follower agents. $\bar{x}_{k}$ represents the position state of the leader $k$ and keeps being a constant.

The dynamic of follower agent $i$ takes the following form:

$$
x_{i}^{(\alpha)}(t)=u_{i}(t), \quad i \in \mathcal{O}
$$

where $x_{i}(t) \in \mathbb{R}^{n}$ is the position state, $u_{i}(t) \in \mathbb{R}^{n}$ is the control input of agent $i$, and $x_{i}^{(\alpha)}(t) \in \mathbb{R}^{n}$ is the $\alpha$ th derivative of $x_{i}(t)$. In practice, the fractional order $\alpha$ often lies in $(0,1]$, so we assume that the order $\alpha$ is a positive real number but not more than 1 in this paper.

For the aforementioned fractional-order dynamics, the following control rule will be used for follower agent $i$ :

$$
u_{i}(t)=\sum_{j \in \mathcal{N}_{i}} a_{i j}\left(x_{j}(t-\tau)-x_{i}(t-\tau)\right)+\sum_{k=N+1}^{N+m} b_{k}^{i}\left(\bar{x}_{k}(t-\tau)-x_{i}(t-\tau)\right),
$$

where $b_{k}^{i} \geq 0, b_{k}^{i}>0$ if and only if the leader $k(k=N+1, N+2, \ldots, N+m)$ is a neighbor of agent $i$. Let $\boldsymbol{B}=\left[\boldsymbol{B}_{N+1}, \boldsymbol{B}_{N+2}, \ldots, \boldsymbol{B}_{N+m}\right]$, where $\boldsymbol{B}_{k} \in \mathbb{R}^{N \times N}$ is a diagonal matrix with $b_{k}^{i}(i=$ $1,2, \ldots, N)$ as its diagonal entry. Assume the time delay in (2.5) satisfies $0<\tau \leq h$. 
Inserting the control rule (2.5) into each follower agent dynamic (2.4), the dynamics of agent $i$ becomes

$$
x^{(\alpha)}(t)=-\left(\mathscr{L} \otimes I_{n}\right) x(t-\tau)+\left[\mathbb{B}\left(I_{m} \otimes \mathbf{1}_{N}\right)\right] \otimes I_{n} \bar{x}(t-\tau),
$$

where $x(t)=\left[x_{1}^{T}(t), x_{2}^{T}(t), \ldots, x_{N}^{T}(t)\right]^{T}, \bar{x}(t)=\left[\bar{x}_{N+1}^{T}(t), \bar{x}_{N+2}^{T}(t), \ldots, \bar{x}_{N+m}^{T}(t)\right]^{T}, \mathscr{H}=\mathcal{L}+$ $B\left(\mathbf{1}_{m} \otimes I_{N}\right)$.

Our objective is to let the $N$ follower agents move into the polytope region formed by the leaders; that is, for any $x_{i}(t)(i=1,2, \ldots, N)$ it can be represented as a convex hull of $\bar{x}_{k}(t)(k=N+1, N+2, \ldots, N+m)$ when $t \rightarrow+\infty$, namely:

$$
\lim _{t \rightarrow+\infty} d_{\Theta}\left(x_{i}(t)\right)=0, \quad i=1,2, \ldots, N
$$

where $\Theta=\left\{\mu \mid \mu \in \operatorname{co}\left\{\bar{x}_{N+1}(t), \bar{x}_{N+2}(t), \ldots, \bar{x}_{N+m}(t)\right\}\right\}$.

\section{Convergence Analysis}

Before starting our main results, we begin with the following lemmas which will play an important role in the proof of main results.

Lemma 3.1. If graph $\overline{\mathcal{G}}$ has a spanning tree, then the matrix $\mathscr{d e}$ associated with $\overline{\mathcal{G}}$ is a positive stable matrix; that is, all the eigenvalues of de lie in the open right hand plane.

Proof. This lemma follows from Lemma 4 in [9] by considering the convex region formed by the leaders as a virtual leader.

Lemma 3.2 (see [25]). The following autonomous system:

$$
\frac{d^{\alpha} x(t)}{d t^{\alpha}}=A x(t), \quad x(0)=x_{0}
$$

with $0<\alpha \leq 1, x \in R^{n}$, and $A \in R^{n \times n}$, is asymptotically stable if and only if $|\arg (\rho(A))|>\alpha \pi / 2$ is satisfied for all eigenvalues of matrix $A$. Also, this system is stable if and only if $|\arg (\rho(A))| \geq \alpha \pi / 2$ is satisfied for all eigenvalues of matrix $A$ with those critical eigenvalues satisfying $|\arg (\rho(A))|=\alpha \pi / 2$ having geometric multiplicity of one. The geometric multiplicity of an eigenvalue $\lambda$ of the matrix $A$ is the dimension of the subspace of vectors $v$ for which $A v=\lambda v$.

When the time delay $\tau=0$, the dynamic network degenerates to the delay-free case, and the dynamic (2.6) can be rewritten as

$$
x^{(\alpha)}(t)=-\left(\mathscr{L} \otimes I_{n}\right) x(t)+\left[B\left(I_{m} \otimes \mathbf{1}_{N}\right)\right] \otimes I_{n} \bar{x}(t) .
$$

Then, we have the following theorem.

Theorem 3.3. For the fractional-order dynamic system (3.2), the follower agents can enter the region spanned by the leaders if the fixed interaction graph $\overline{\mathcal{G}}$ has a directed spanning tree. 
Proof. By Lemma 3.1, we learn that matrix $\mathscr{\ell}$ is invertible. Let $\delta(t)=x(t)-\left[\mathscr{\ell}^{-1} \boldsymbol{B}\left(I_{m} \otimes \mathbf{1}_{N}\right)\right] \otimes$ $\mathbf{I}_{n} \bar{x}(t)$. Then, we get the following error system:

$$
\delta^{(\alpha)}(t)=-\left(\mathscr{\ell} \otimes I_{n}\right) \delta(t)
$$

Since all the eigenvalues of matrix $\mathscr{H}$ lie in the open right hand plane, then, $\mid \arg (-\mathscr{\ell})) \mid \in$ $(\pi / 2, \pi]$ and $2 \arg (\rho(\mathscr{\ell})) / \pi>1$ hold. It follows from Lemma 3.2 that system (3.3) is asymptotically stable for any $\alpha \in(0,1]$.

In the sequel, we will prove that all the follower agents can be aggregated in a polytope region formed by the leaders. In other words, we need only to prove that for any vector $\bar{x}_{i}^{*}(t) \in \mathbb{R}^{n}(i=1,2, \ldots, N)$, the region can be expressed as a convex hull of $\bar{x}_{k}(t) \in \mathbb{R}^{n}(k=N+$ $1, N+2, \ldots, N+m)$. This problem can be transformed to prove that matrix $\left[\mathscr{L}^{-1} \mathcal{B}\left(I_{m} \otimes \mathbf{1}_{N}\right)\right] \otimes I_{n}$ is a row stochastic matrix that is, it is a nonnegative matrix, and the sum of the items in every row is 1 .

Since all the eigenvalues of $\mathscr{t}$ have positive real parts, there exist a positive scalar $\kappa>0$ and nonnegative matrix $Z$ such that $\mathscr{H}=\kappa I-Z$ holds. Obviously, $\mathcal{\kappa}>\rho(Z)$ and $\lambda_{i}(\mathscr{\ell})=\kappa-\lambda_{i}(Z), \forall i=1,2, \ldots, N$. Therefore,

$$
\mathscr{H}^{-1}=(\kappa I-Z)^{-1}=\frac{1}{\mathcal{\kappa}}\left(I+\frac{1}{\mathcal{K}} Z+\frac{1}{\mathcal{K}^{2}} Z^{2}+\cdots\right) \geq 0 .
$$

It can be seen that matrix $\mathscr{L}^{-1} \otimes I_{n}$ is a nonnegative matrix and so is $\left[\mathscr{L}^{-1} \mathcal{B}\left(I_{m} \otimes \mathbf{1}_{N}\right)\right] \otimes I_{n}$. Notice that $\mathscr{H}=L+B\left(\mathbf{1}_{m} \otimes I_{N}\right)$ and $L \mathbf{1}_{N}=0$; we obtain

$$
\begin{aligned}
\left(\mathscr{\ell} \otimes I_{n}\right)\left(\mathbf{1}_{N} \otimes \mathbf{1}_{n}\right) & =\left(\left(L+B\left(\mathbf{1}_{m} \otimes I_{N}\right)\right) \otimes I_{n}\right)\left(\mathbf{1}_{N} \otimes \mathbf{1}_{n}\right) \\
& =\left(\sum_{i=N+1}^{N+m} B_{i} \mathbf{1}_{N}\right) \otimes \mathbf{1}_{n} .
\end{aligned}
$$

Thus,

$$
\begin{aligned}
\left(\left[\mathscr{L}^{-1} \mathcal{B}\left(I_{m} \otimes \mathbf{1}_{N}\right)\right] \otimes I_{n}\right)\left(\mathbf{1}_{m} \otimes \mathbf{1}_{n}\right) & =\left(\left(\mathscr{H} \otimes I_{n}\right)^{-1}\left[\mathcal{B}\left(I_{m} \otimes \mathbf{1}_{N}\right)\right] \otimes I_{n}\right)\left(\mathbf{1}_{m} \otimes \mathbf{1}_{n}\right) \\
& =\left(\mathscr{H} \otimes I_{n}\right)^{-1}\left(\sum_{i=N+1}^{N+m} B_{i} \mathbf{1}_{N}\right) \otimes \mathbf{1}_{n} \\
& =\mathbf{1}_{N} \otimes \mathbf{1}_{n} .
\end{aligned}
$$

It is obvious that $\left[\mathscr{L}^{-1} \mathcal{B}\left(I_{m} \otimes \mathbf{1}_{N}\right)\right] \otimes I_{n}$ is a row stochastic matrix. Thus, the conclusion of Theorem 3.3 holds.

Remark 3.4. This result coincides with the existing results in [26] and has been extended to fractional order cases.

In what follows, we will focus on the convergence analysis of (2.6) under fixed and directed interconnection topologies in delayed communication channels. 
Theorem 3.5. For the fractional-order dynamic system (2.6), the follower agents can enter the region spanned by the leaders if the fixed interaction graph $\overline{\mathcal{G}}$ has a directed spanning tree and $\tau<\tau^{*}$, where

$$
\tau^{*}=\min _{i \in \supset} \frac{\pi-(\alpha \pi / 2)+\eta_{i}}{\left(\left|\mu_{i}\right|\right)^{1 / \alpha}}
$$

$\mu_{i}, i \in \supset$ is the ith eigenvalue of matrix $\mathscr{\ell}, \eta_{i}=\arctan \left(\operatorname{Im}\left(\mu_{i}\right) / \operatorname{Re}\left(\mu_{i}\right)\right)$.

Proof. Since the communication topology $\overline{\mathcal{G}}$ has a spanning tree, all the eigenvalues of $\mathscr{H}$ have positive real parts by Lemma 3.1. Therefore, matrix $\mathscr{L}$ is invertible. Let $\delta(t)=x(t)-$ $x^{*}(t), x^{*}(t)=\left[\mathscr{L}^{-1} \mathcal{B}\left(I_{m} \otimes \mathbf{1}_{N}\right)\right] \otimes I_{n} \bar{x}(t)$. Then, we get the following error system

$$
\delta^{(\alpha)}(t)=-\left(\mathscr{\ell} \otimes I_{n}\right) \delta(t-\tau) .
$$

Taking Laplace transform of system (3.8), it yields that

$$
s^{\alpha} \xi(s)-s^{\alpha-1} \xi(0)=-e^{-\tau s}\left(\mathscr{L} \otimes I_{n}\right) \xi(s),
$$

where $\xi(s)$ is the Laplace transforms of position states $\delta(t)$. After some simple manipulation we obtain

$$
\xi(s)=(\Delta(s))^{-1} \xi(0),
$$

where $\Delta(s)=s^{\alpha} I+e^{-\tau s}\left(\mathscr{L} \otimes I_{n}\right)$, which is called a characteristic matrix in [27]. The distribution of $\operatorname{det}(\Delta(s))$ 's eigenvalues totally determines the stability of system (3.8). Thus, a sufficient condition for the error dynamics (3.8) which converges to zero is that all the poles of $\Delta(s)$ are located at the open left half plane or $s=0$.

Then, the characteristic equation of (3.8) is

$$
s^{\alpha}+e^{-\tau s} \mu_{i}=0,
$$

where $\mu_{i}$ is the $i$ th eigenvalue of matrix $\mathscr{d}$.

Obviously, $s \neq 0$. Now, we turn to prove that the poles of (3.11) are located at the open left half plane. Since the directed graph $\overline{\mathcal{G}}$ has a spanning tree, all the eigenvalues of $\mathscr{H}$ have positive real parts. Without loss of generality, we assume that the eigenvalues satisfy $0<$ $\operatorname{Re}\left(\mu_{1}\right) \leq \operatorname{Re}\left(\mu_{2}\right) \leq \cdots \leq \operatorname{Re}\left(\mu_{n}\right)$. Based on the Nyquist stability theorem, all the roots of (3.11) lie in the open left half complex plane, if and only if the Nyquist curve $e^{-\tau s} \mu_{i} / s^{\alpha}$ does not enclose the point $(-1,0 \mathbf{i})$ for any $w \in \mathbb{R}$, where $\mathbf{i}$ is the imaginary unit. we have

Therefore, assume that $s=\mathbf{i} w=w(\cos (\pi / 2)+\mathbf{i} \sin (\pi / 2))(w>0)$ is a root of (3.11);

$$
w^{\alpha}\left(\cos \frac{\alpha \pi}{2}+\mathbf{i} \sin \frac{\alpha \pi}{2}\right)+\left|\mu_{i}\right|\left(\cos \left(\eta_{i}-w \tau\right)+\mathbf{i} \sin \left(\eta_{i}-w \tau\right)\right)=0
$$


where $\eta_{i}=\arctan \left(\operatorname{Im}\left(\mu_{i}\right) / \operatorname{Re}\left(\mu_{i}\right)\right)$. Separating the real and imaginary parts of (3.12) yields

$$
\begin{gathered}
w^{\alpha} \cos \frac{\alpha \pi}{2}+\left|\mu_{i}\right| \cos \left(\eta_{i}-w \tau\right)=0 \\
w^{\alpha} \sin \frac{\alpha \pi}{2}+\left|\mu_{i}\right| \sin \left(\eta_{i}-w \tau\right)=0 .
\end{gathered}
$$

From the above two equations, one gets

$$
w^{2 \alpha}+\left|\mu_{i}\right|^{2}+2\left|\mu_{i}\right| w^{\alpha} \cos \left(\frac{\alpha \pi}{2}-\eta_{i}+w \tau\right)=0,
$$

that is

$$
\left(w^{\alpha}-\left|\mu_{i}\right|\right)^{2}+2\left|\mu_{i}\right| w^{\alpha}\left[1+\cos \left(\frac{\alpha \pi}{2}-\eta_{i}+w \tau\right)\right]=0
$$

Obviously, the two terms in the left-hand side of (3.15) are nonnegative the equality holds if and only if both the two terms are zero, namely:

$$
\begin{gathered}
w^{\alpha}=\left|\mu_{i}\right|, \\
1+\cos \left(\frac{\alpha \pi}{2}-\eta_{i}+w \tau\right)=0 .
\end{gathered}
$$

Thus, combining (3.16) and (3.17), we can easily obtain that

$$
\pi+2 k \pi=\left(\left|\mu_{i}\right|\right)^{1 / \alpha} \tau+\frac{\alpha \pi}{2}-\eta_{i}, \quad k=0,1,2, \ldots
$$

Therefore, the smallest time-delay $\tau>0$ occurs at $k=0$ and satisfies

$$
\tau=\frac{\pi-(\alpha \pi / 2)+\eta_{i}}{\left(\left|\mu_{i}\right|\right)^{1 / \alpha}} .
$$

Similarly, one can repeat the very argument for the case that $w<0$ and get similar conclusion.

Thus, the Nyquist plot of $e^{-\tau s} \mu_{i} / s^{\alpha}$ does not enclose the point $(-1,0 \mathbf{i})$ for all $i>1$ if

$$
\tau<\frac{\pi-(\alpha \pi / 2)+\eta_{i}}{\left(\left|\mu_{i}\right|\right)^{1 / \alpha}} .
$$

Then all the roots of $\operatorname{det}(\Delta(s))$ lie in the open left hand plan. Therefore, the error system (3.8) is asymptotically stable, that is, $x \rightarrow x^{*}=\left[\mathscr{\ell}^{-1} \boldsymbol{B}\left(I_{m} \otimes \mathbf{1}_{N}\right)\right] \otimes I_{n} \bar{x}$, as $t \rightarrow+\infty$. The rest of the proof is similar to that of Theorem 3.3 and hence is omitted.

For the case of the considered undirected graph, that is, agent $i$ and agent $j$ can receive information from each other when there exists an edge between $i$ and $j$; we can get the following result. 


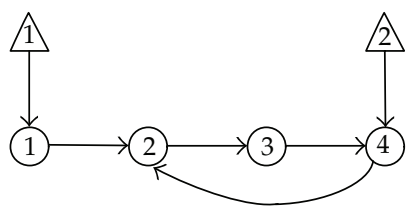

Figure 1: Directed graph $\mathcal{G}_{1}$ and $\overline{\mathcal{G}}_{1}$.

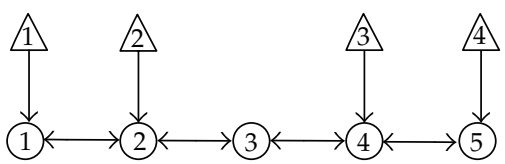

Figure 2: Directed graph $\mathcal{G}_{2}$ and $\overline{\mathcal{G}}_{2}$.

Theorem 3.6. Under an undirected time-invariant interaction graph $\overline{\mathcal{G}}$, that is, strongly connected, the follower agents can enter the region spanned by the leaders if $\tau<\bar{\tau}^{*}$, where

$$
\bar{\tau}^{*}=\min _{i \in \supset, \mu_{i}>0} \frac{\pi-(\alpha \pi / 2)}{\left(\left|\mu_{i}\right|\right)^{1 / \alpha}}
$$

$\mu_{i}, i \in \supset$ is the ith eigenvalue of matrix $\mathcal{L}$.

Proof. The proof of Theorem 3.6 is similar to that of the Theorem 3.5 by noting that the eigenvalues of $\mathscr{t}$ are nonnegative real number, and hence omitted.

\section{Numerical Examples}

In this section, two numerical simulations will be presented to illustrate the effectiveness of the theoretical results obtained in the previous sections. In all the simulations, all dynamics of agent are integrated with a fixed time step 0.05 . The following two directed graphs with $0-1$ weights will be needed in the analysis of this section. Circle and triangle stand for follower agent and leader, respectively.

Example 4.1. Consider a dynamic fractional-order network of four follower agents and two leaders with a fixed topology given in Figure 1. Obviously, the topology $\overline{\mathcal{G}}_{1}$ has a spanning tree. Suppose that all the agents are moving in a horizontal line and each follower agent can receive the state information of its neighbors precisely. The matrix $\mathscr{H}$ is $\left(\begin{array}{cccc}2 & -1 & 0 & 0 \\ 0 & 2 & -1 & -1 \\ 0 & 0 & 1 & -1 \\ 0 & 0 & 0 & 1\end{array}\right)$, and its four eigenvalues are $0.2451,1.0000,1.8774+0.7449 \mathbf{i}, 1.8774-0.7449 \mathbf{i}$, respectively. The initial position states of follower agents are generated randomly in $[1,6]$, and leaders are chosen as $\bar{x}_{1}(0)=0.5, \bar{x}_{2}(0)=-0.5$. Let $\alpha=0.92$ and $u_{0}(t)=0.2$. The state trajectories of the fractionalorder close-loop systems (2.6) are shown in Figure 3. It can be easily seen that as time goes on, the whole follower agents will be flocked in the segment $[-0.5,0.5]$, which is formed by the two leaders. 


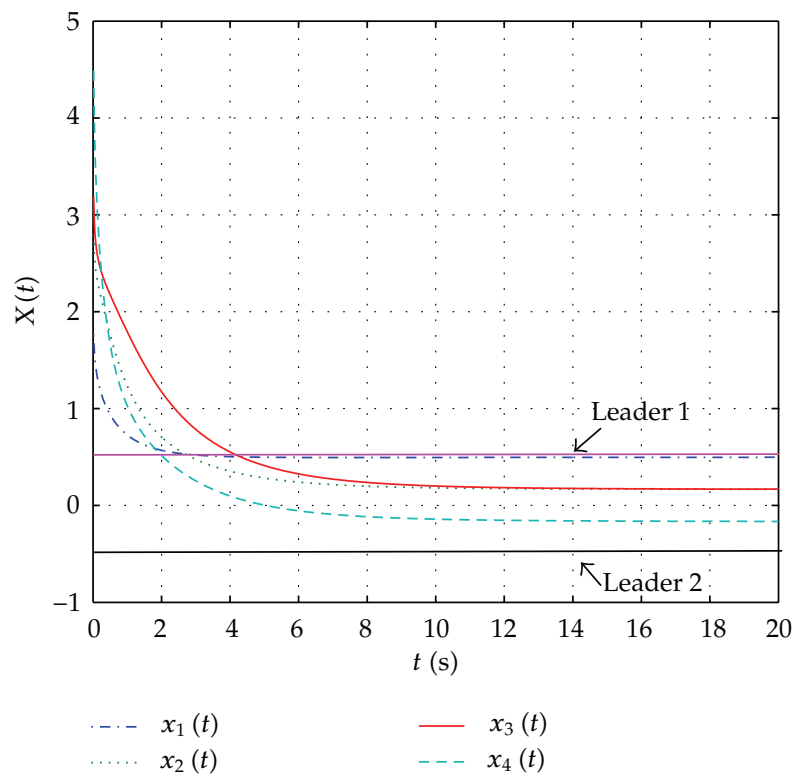

Figure 3: State trajectories of four follower agents and two leaders under the topology $\overline{\mathcal{G}}_{1}$.

Example 4.2. Consider a dynamic fractional-order network of five follower agents and four leaders moving in a plane. The interconnection topology among the above agents is given in Figure 2. Clearly, $\overline{\mathcal{G}}_{2}$ is strongly connected. The matrix $\mathscr{d}$ is

$$
\left(\begin{array}{ccccc}
2 & -1 & 0 & 0 & 0 \\
-1 & 3 & -1 & 0 & 0 \\
0 & -1 & 2 & -1 & 0 \\
0 & 0 & -1 & 3 & -1 \\
0 & 0 & 0 & -1 & 2
\end{array}\right)
$$

and its five eigenvalues are $0.6972,1.3820,2.0000,3.6180,4.3028$, respectively. The initial position states of agents and leaders are given as follows:

$$
\begin{gathered}
x_{1}(0)=(0,2)^{T}, \quad x_{2}(0)=(0,3)^{T}, \quad x_{3}(0)=(0,4)^{T}, \quad x_{4}(0)=(0,5)^{T}, \quad x_{5}(0)=(0,6)^{T}, \\
\bar{x}_{1}(0)=(4,5)^{T}, \quad \bar{x}_{2}(0)=(5,6)^{T}, \quad \bar{x}_{3}(0)=(6,5)^{T}, \quad \bar{x}_{4}(0)=(5,4)^{T} .
\end{gathered}
$$

Take the fractional order $\alpha=0.96$ in (2.1). Then, it can be seen from Theorem 3.6 that all the followers will enter into the region formed by the leaders if $\tau<0.3573$. Figures 4 and 5 depict the simulation results for position trajectories under different time delays. From these simulations, one can easily find that the containment control is realized via protocol (2.5) as long as the time delay doesnot exceed the upper bound. These simulations are consistent with the theoretical result in Section 3. 


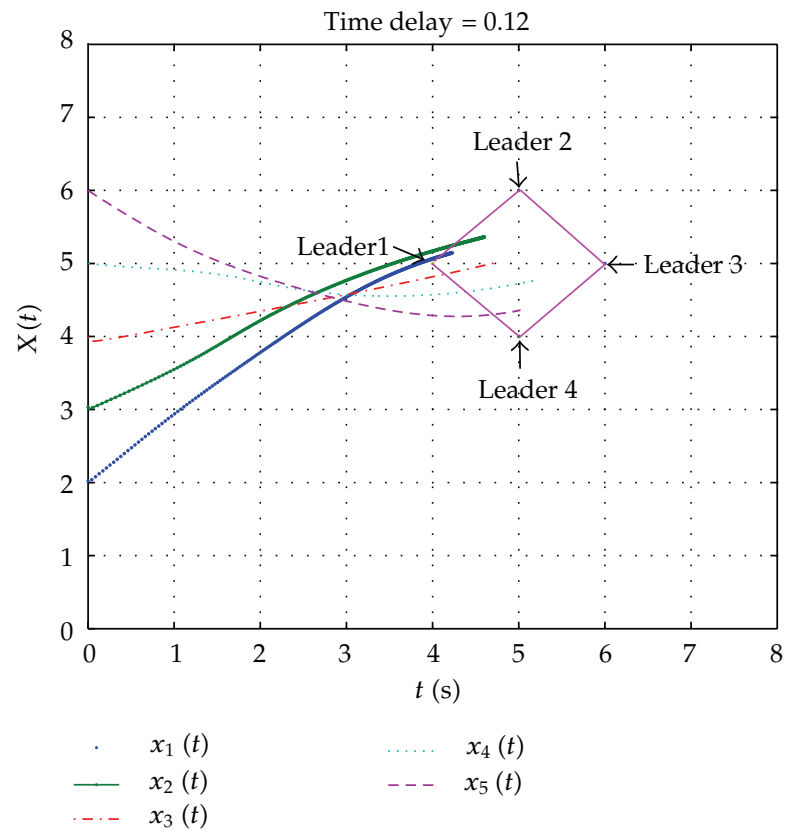

Figure 4: State trajectories of five follower agents and four leaders under the topology $\overline{\mathcal{G}}_{2}$ and time delay $\tau=0.12$.

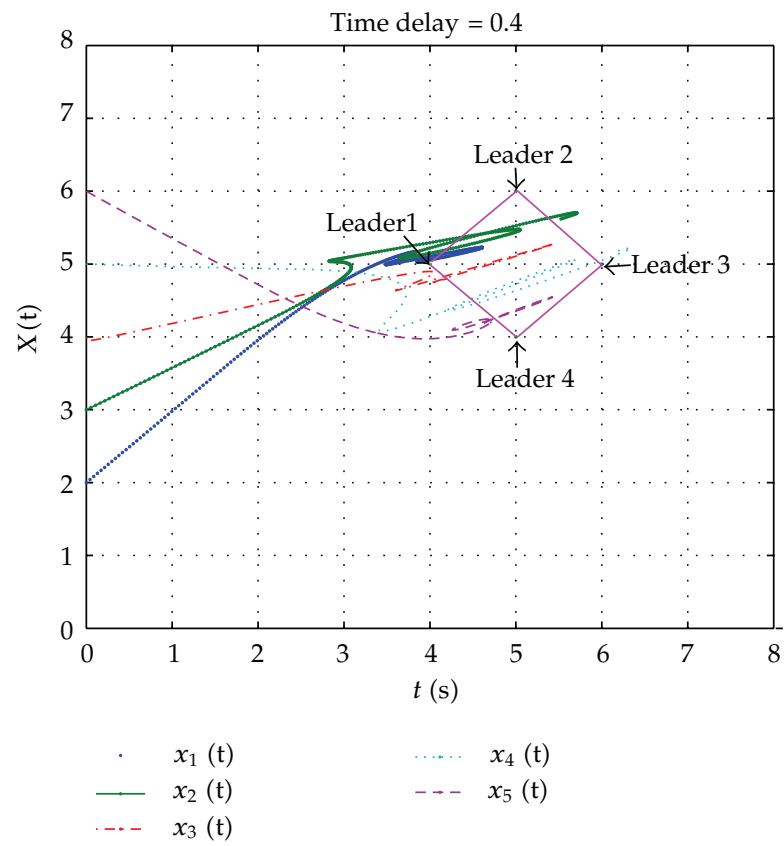

Figure 5: State trajectories of five follower agents and four leaders under the topology $\overline{\mathcal{G}}_{2}$ and time delay $\tau=0.4$. 


\section{Conclusion}

In this paper, the containment control problem of multiple leaders has been considered for networks of fractional-order dynamics with delay-dependent communication channels. Utilizing algebraic graph theory, matrix theory, Nyquist stability theorem, and frequency domain method, some sufficient conditions are obtained. It is shown that all the follower agents will ultimately move into the convex hull which is spanned by the leaders, for appropriate communication time delay if the topology of weighted network has a spanning tree. Moreover, two numerical simulations are provided to validate the effectiveness of our theoretical analysis.

\section{Acknowledgments}

This work was supported by the National Natural Science Foundation of China under Grant no. 61174070, the Specialized Research Found for the Doctoral Program no. 20110172110033, the NSFC-Guangdong Joint Foundation Key Project under Grant no. U0735003, the Oversea Cooperation Foundation under Grant 60828006 and the Key Laboratory of Autonomous Systems and Network Control, Ministry of Education.

\section{References}

[1] F. Xiao, L. Wang, J. Chen, and Y. Gao, "Finite-time formation control for multi-agent systems," Automatica, vol. 45, no. 11, pp. 2605-2611, 2009.

[2] R. Vidal, O. Shakernia, and S. Sastry, "Formation control of nonholonomic mobile robots with omnidirectional visual servoing and motion segmentation," in Proceedings of the IEEE Conference on Robotics and Automation (ICRA '03), pp. 584-589, Taipei, Taiwan, September 2003.

[3] J. A. Fax and R. M. Murray, "Information flow and cooperative control of vehicle formations," IEEE Transactions on Automatic Control, vol. 49, no. 9, pp. 1465-1476, 2004.

[4] F. Cucker and S. Smale, "Emergent behavior in flocks," IEEE Transactions on Automatic Control, vol. 52, no. 5, pp. 852-862, 2007.

[5] R. Olfati-Saber, "Flocking for multi-agent dynamic systems: algorithms and theory," IEEE Transactions on Automatic Control, vol. 51, no. 3, pp. 401-420, 2006.

[6] L. Xiao, S. Boyd, and S. Lall, "A scheme for robust distributed sensor fusion based on average consensus," in Proceedings of the 4th International Conference on Information Process in Sensor Networks, pp. 63-70, Los Angeles, Calif, USA, April 2005.

[7] R. Saber and J. Shamma, "Consensus filters for sensor networks and distributed sensor fusion," in Proceedings of the 44th IEEE Conference on Decision and Control, and the European Control Conference (CDCECC '05), pp. 6698-6703, Seville, Spain, 2005.

[8] T. Li and J. Zhang, "Mean square average-consensus under measurement noise and fixed topologies: necessary and sufficient conditions," Automatica, vol. 45, no. 8, pp. 1929-1936, 2009.

[9] J. Hu and Y. Hong, "Leader-following coordination of multi-agent systems with coupling time delays," Physica A, vol. 374, no. 2, pp. 853-863, 2007.

[10] W. Ren, R. W. Beard, and D. Kingston, "Multi-agent kalman consensus with relative uncertainty," in Proceedings of the American Control Conference, pp. 1865-1870, Portland, Ore, USA, June 2005.

[11] R. Olfati-Saber and R. M. Murray, "Consensus problems in networks of agents with switching topology and time-delays," IEEE Transactions on Automatic Control, vol. 49, no. 9, pp. 1520-1533, 2004

[12] W. Yu, G. Chen, and J. Cao, "Adaptive synchronization of uncertain coupled stochastic complex networks," Asian Journal of Control, vol. 13, no. 3, pp. 418-429, 2011.

[13] M. Barahona and L. Pecora, "Synchronization in small-world systems," Physical Review Letters, vol. 89, no. 5, Article ID 054101-4, 2002

[14] N. A. Lynch, Distributed Algorithms, The Morgan Kaufmann Series in Data Management Systems, Morgan Kaufmann, San Francisco, Calif, USA, 1996. 
[15] W. Ren and R. W. Beard, Distributed Consensus in Multi-Vehicle Cooperative Control, Springer, London, UK, 2008.

[16] M. Cao, A. S. Morse, and B. D. O. Anderson, "Agreeing asynchronously," IEEE Transactions on Automatic Control, vol. 53, no. 8, pp. 1826-1838, 2008.

[17] I. Podlubny, Fractional Differential Equations: An Introduction to Fractional Derivatives, Fractional Differential Equations, to Methods of Their Solution and Some of Their Applications, vol. 198 of Mathematics in Science and Engineering, Academic Press, New York, NY, USA, 1999.

[18] K. Oldham and J. Spanier, The Fractional Calculus, Academic Press, New York, NY, USA, 1974.

[19] Y. Cao, Y. Li, W. Ren, and Y. Chen, "Distributed coordination algorithms for multiple fractionalorder systems," in Proceedings of the IEEE Conference on Decision and Control (CDC '08), pp. 2920-2925, Cancun, Mexico, December 2008.

[20] Y. Cao, Y. Li, W. Ren, and Y. Chen, "Distributed coordination of networked fractional-order systems," IEEE Transactions on Systems, Man, and Cybernetics B, vol. 40, no. 2, pp. 362-370, 2010.

[21] W. Sun, Y. Li, C. Li, and Y. Chen, "Convergence speed of a fractional order consensus algorithm over undirected scale-free networks," Asian Journal of Control, vol. 13, no. 6, pp. 936-946, 2011.

[22] J. Shen, J. D. Cao, and J. Lu, "Consenus of fractional-order systems with non-uniform input and communication delays," Journal of Systems and Control Engineering, vol. 226, no. 2, pp. 271-283, 2012.

[23] J. Shen and J. Cao, "Necessary and sufficient conditions for consenus of delayed fractional-order systems over directed graph," 2011, http://www.paper.edu.cn/.

[24] Y. Cao and W. Ren, "Distributed formation control for fractional-order systems: dynamic interaction and absolute/relative damping," Systems E Control Letters, vol. 59, no. 3-4, pp. 233-240, 2010.

[25] D. Matignon, "Stability result on fractional differential equations with applications to control processing," in Proceedings of the IMACSSMC, pp. 963-968, Lille, France, 1996.

[26] Y. Cao and W. Ren, Distributed Coordination of Multi-agent Networks Emergent Problems, Models, and Issues, Springer, London, UK, 2011.

[27] W. Deng, C. Li, and J. Lü, "Stability analysis of linear fractional differential system with multiple time delays," Nonlinear Dynamics, vol. 48, no. 4, pp. 409-416, 2007. 


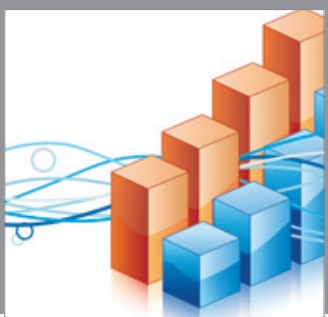

Advances in

Operations Research

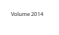

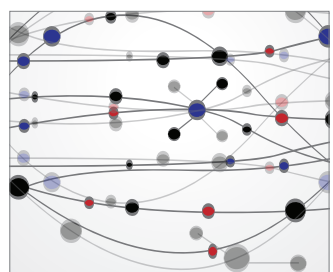

\section{The Scientific} World Journal
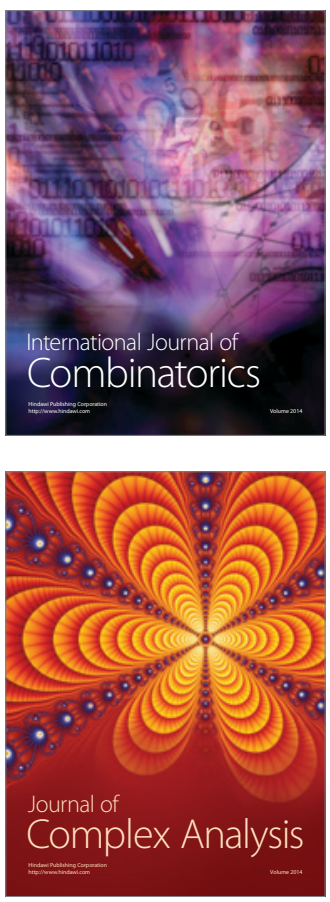

International Journal of

Mathematics and

Mathematical

Sciences
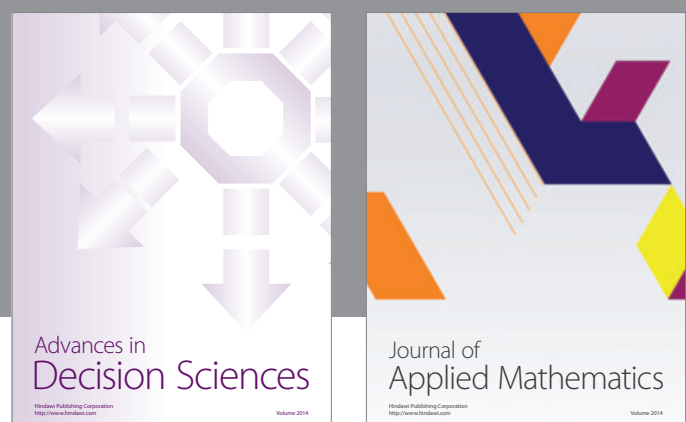

Journal of

Applied Mathematics
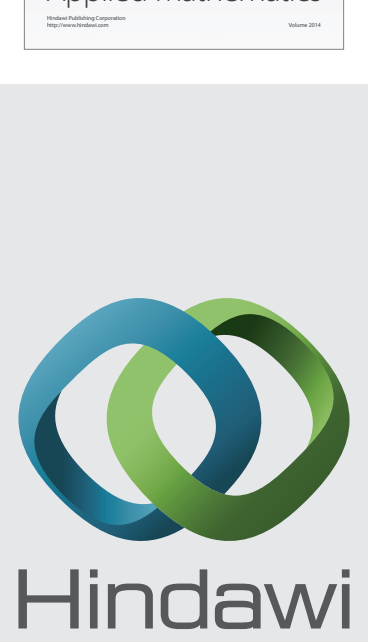

Submit your manuscripts at http://www.hindawi.com
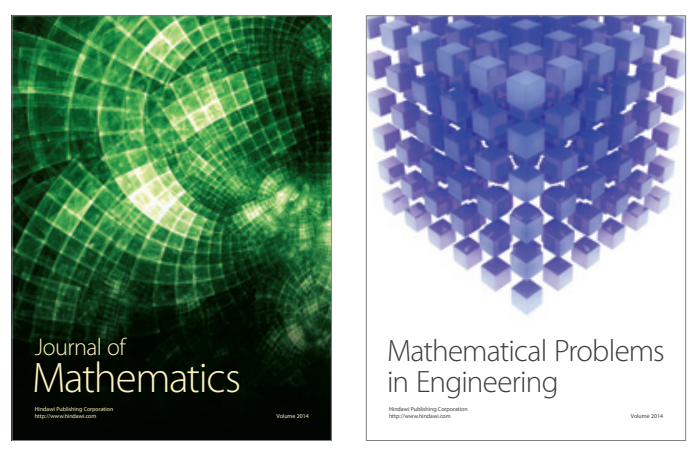

Mathematical Problems in Engineering
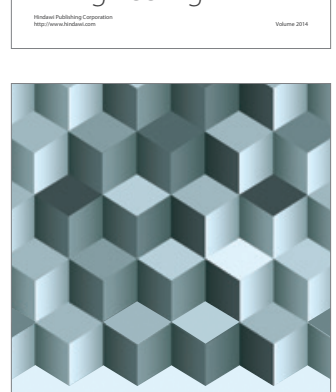

Journal of

Function Spaces
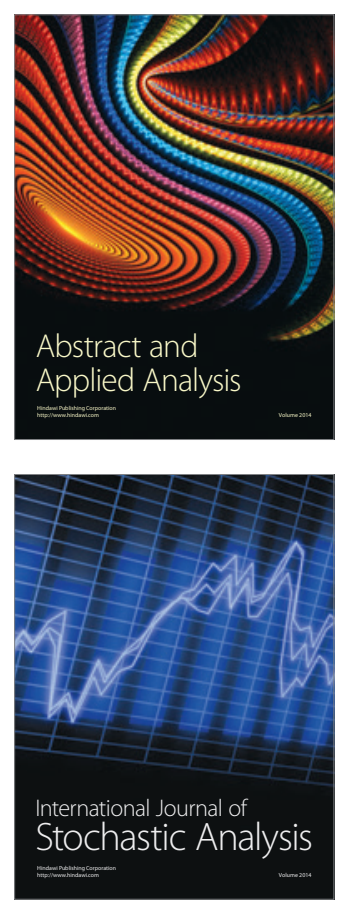

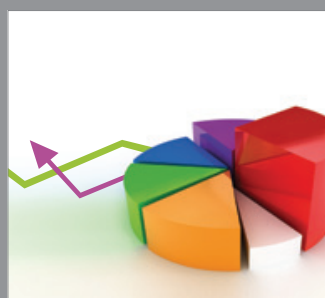

ournal of

Probability and Statistics

Promensencen
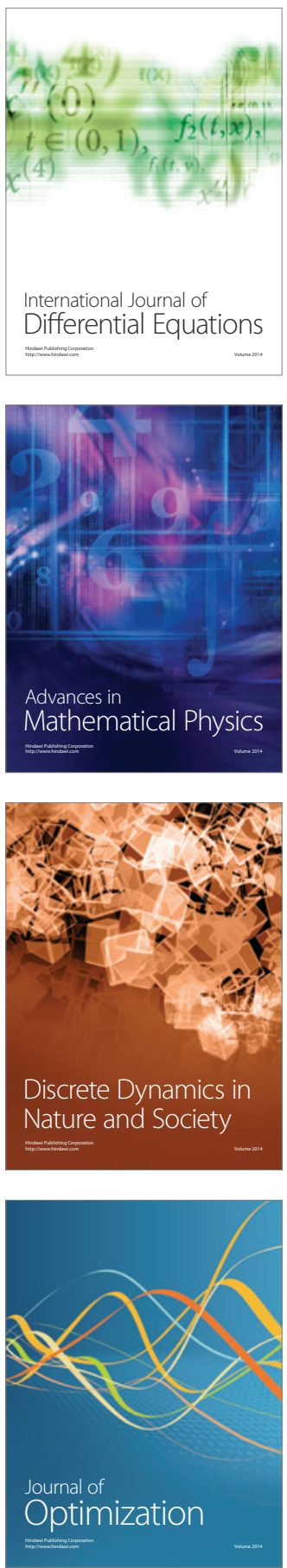